For mat $i$ on of el ectron i nt ernal transport barrier and achi evement of high i on temper at ure in Lar ge Hel i cal Devi ce

\begin{tabular}{|l|l|}
\hline $\begin{array}{l}\text { j our nal or } \\
\text { publ i cat i on } \mathrm{titl} \text { e }\end{array}$ & Physi cs of Pl asmas \\
\hline vol une & Vol . 10 \\
\hline number & I ssue 5 \\
\hline page r ange & pp. 1788 1795 \\
\hline year & 2003 $05-01$ \\
\hline URL & ht t p: //hdl . handl e. net /10655/3882 \\
\hline
\end{tabular}




\title{
Formation of electron internal transport barrier and achievement of high ion temperature in Large Helical Device ${ }^{\text {a) }}$
}

\author{
Y. Takeiri, ${ }^{\text {b) }}$ T. Shimozuma, S. Kubo, S. Morita, M. Osakabe, O. Kaneko, K. Tsumori, \\ Y. Oka, K. Ikeda, K. Nagaoka, N. Ohyabu, K. Ida, M. Yokoyama, J. Miyazawa, M. Goto, \\ K. Narihara, I. Yamada, H. Idei, Y. Yoshimura, N. Ashikawa, M. Emoto, H. Funaba, \\ S. Inagaki, M. Isobe, K. Kawahata, K. Khlopenkov, T. Kobuchi, A. Komori, A. Kostrioukov, \\ R. Kumazawa, Y. Liang, S. Masuzaki, T. Minami, T. Morisaki, S. Murakami, S. Muto, \\ T. Mutoh, Y. Nagayama, Y. Nakamura, H. Nakanishi, Y. Narushima, K. Nishimura, N. Noda, \\ S. Ohdachi, T. Ozaki, B. J. Peterson, A. Sagara, K. Saito, S. Sakakibara, R. Sakamoto, \\ M. Sasao, K. Sato, M. Sato, T. Seki, M. Shoji, H. Suzuki, N. Tamura, K. Tanaka, K. Toi, \\ T. Tokuzawa, K. Y. Watanabe, T. Watari, Y. Xu, H. Yamada, M. Yoshinuma, K. Itoh, \\ K. Ohkubo, T. Satow, S. Sudo, T. Uda, K. Yamazaki, Y. Hamada, K. Matsuoka, \\ O. Motojima, and M. Fujiwara \\ National Institute for Fusion Science, Toki 509-5292, Japan
}

T. Notake, N. Takeuchi, Y. Torii, S. Yamamoto, and T. Yamamoto Department of Energy Engineering and Science, Nagoya University, Nagoya 464-8603, Japan

T. Akiyama

Research Laboratory for Nuclear Reactors, Tokyo Institute of Technology, Tokyo 152-8550, Japan

P. Goncharov and T. Saida

Department of Fusion Science, School of Mathematical and Physical Science, Graduate University for Advanced Studies, Hayama 240-0193, Japan

H. Kawazome

Graduate School of Energy Science, Kyoto University, Uji 611-0011, Japan

H. Nozato

Graduate School of Frontier Sciences, The University of Tokyo, Tokyo 113-0033, Japan

(Received 11 November 2002; accepted 20 January 2003)

\begin{abstract}
An internal transport barrier (ITB) was observed in the electron temperature profile in the Large Helical Device [O. Motojima et al., Phys. Plasmas 6, 1843 (1999)] with a centrally focused intense electron cyclotron resonance microwave heating. Inside the ITB the core electron transport was improved, and a high electron temperature, exceeding $10 \mathrm{keV}$ in a low density, was achieved in a collisionless regime. The formation of the electron-ITB is correlated with the neoclassical electron root with a strong radial electric field determined by the neoclassical ambipolar flux. The direction of the tangentially injected beam-driven current has an influence on the electron-ITB formation. For the counter-injected target plasma, a steeper temperature gradient, than that for the co-injected one, was observed. As for the ion temperature, high-power NBI (neutral beam injection) heating of 9 MW has realized a central ion temperature of $5 \mathrm{keV}$ with neon injection. By introducing neon gas, the NBI absorption power was increased in low-density plasmas and the direct ion heating power was much enhanced with a reduced number of ions, compared with hydrogen plasmas. (C) 2003 American Institute of Physics. [DOI: 10.1063/1.1560613]
\end{abstract}

\section{INTRODUCTION}

The Large Helical Device (LHD) is the world's largest superconducting helical system with an $l=2 / \mathrm{m}=10$ field period, of which the plasma major radius is $3.42-4.2 \mathrm{~m}$ and the average minor radius is about $0.6 \mathrm{~m} \cdot{ }^{1,2}$ Four years of operation of LHD has revealed experimentally several physical issues on the toroidal plasma confinement in addition to the intrinsic advantages of helical systems such as steady-state operation without current drive, absence of disruption, and

\footnotetext{
${ }^{\text {a)}}$ Paper BI1 4, Bull. Am. Phys. Soc. 47, 19 (2002).

b)Invited speaker. Electronic mail: takeiri@nifs.ac.jp
}

no feedback stabilization. ${ }^{3,4}$ In an inward shifted configuration of the magnetic axis $\left(R_{\mathrm{ax}}\right)$ of $3.6 \mathrm{~m}$ from the standard one of $R_{\mathrm{ax}}=3.75 \mathrm{~m}$, the energy confinement time is 1.5 times longer than that predicted by the ISS95 (international stellarator scaling 95$),{ }^{5}$ owing to enhanced confinement in the core region, in addition to the high edge pressure observed commonly in both axes. ${ }^{6,7}$ The volume-averaged beta, $\langle\beta\rangle$, also exceeded $3 \%$ in the inward shifted configuration of $R_{\text {ax }}=3.6 \mathrm{~m}$, where theoretically the ideal linear magnetohydrodynamic (MHD) mode (interchange mode) should be unstable due to the presence of the magnetic hill region. ${ }^{8}$ This compatibility of enhanced confinement and MHD stability in the inward shifted configuration is one of the most significant 
physical results of LHD experiments to date. ${ }^{9}$ Moreover, the high-energy particles are well confined in the inward shifted configuration, which is confirmed with the successful ICRF (ion cyclotron range of frequency) heating experiments. ${ }^{10-12}$ Together with the demonstration of long-period plasma sustainment exceeding $1 \mathrm{~min}$, in which the plasma confinement characteristics are almost the same as those in the short-pulse discharges, ${ }^{13-15}$ these physics achievements lead to further understanding of toroidal plasmas including tokamaks, as well as indicate a high potential of the helical systems as a fusion reactor comparable to the tokamaks.

On the other hand, the neoclassical theory predicts reduced losses in the collisionless or long-mean-free-path regime for stellarators with radial electric field. Although improvement of the thermal transport has been reported in the presence of radial electric field in medium-sized helical system devices, ${ }^{16,17}$ a high-temperature plasma relevant to the fusion reactor should be achieved in a large-scaled helical system device like LHD. In the previous experimental campaign of LHD, a high electron temperature of $10 \mathrm{keV}$ was achieved with a centrally focused intense ECR (electron cyclotron resonance) microwave, ${ }^{18}$ and an internal transport barrier (ITB) was observed in the electron temperature profile when the ECR microwave was superposed on the neutral-beam (NB) heated plasmas. ${ }^{19}$ As for the ion temperature, high power NBI (neutral beam injection) heating of 9 MW realized a central ion temperature of $5 \mathrm{keV}$ with neon injection. $^{20,21}$

In this article, the electron-ITB observed in LHD is discussed from the viewpoints of transport improvement and the role of the electric field, and the mechanism of the ion temperature rise with neon injection is also investigated. After a brief summary of LHD and the heating system, in Sec. III characteristics of the electron-ITB and the influence of the injection direction of the NBI on the ITB profile shape are presented, and conditions for the transition to the electron root are discussed compared with the ECR heated plasmas without NBI. The achievement of high ion temperature with neon injection is described in Sec. IV, followed by the summary.

\section{LARGE HELICAL DEVICE AND HEATING SYSTEM}

A superconducting coil system of the LHD consists of a pair of continuous helical coils and three pairs of poloidal coils, which realize a variety of the magnetic geometries. The magnetic axis position $\left(R_{\mathrm{ax}}\right)$ achieved experimentally ranges $3.42-4.2 \mathrm{~m}$ controlled by the vertical field coils. In the inward shifted position $\left(R_{\mathrm{ax}} \leqslant 3.6 \mathrm{~m}\right)$ from the standard one $\left(R_{\mathrm{ax}}=3.75 \mathrm{~m}\right)$, the neoclassical transport and the fast ion confinement are improved although the linear ideal MHD mode (interchange mode) should be destabilized due to a magnetic hill configuration, and vice versa in the outward shifted one. The magnetic field strength on the axis $\left(B_{\mathrm{ax}}\right)$ achieved is $2.976 \mathrm{~T}$ at $R_{\mathrm{ax}}=3.5 \mathrm{~m}$, and gradually decreases as $R_{\mathrm{ax}}$ shifts outward, keeping the mod- $B$ structure.

The electron cyclotron resonance heating (ECRH) system employs three $168 \mathrm{GHz}$, two $84 \mathrm{GHz}$, and two $82.7 \mathrm{GHz}$ gyrotrons, and each microwave is injected on the equatorial plane as a strongly focused Gaussian beam using vertical and horizontal antenna systems with quasi-optical mirrors. ${ }^{22}$ The total injection power achieved was $1.9 \mathrm{MW}$, and the injected microwaves can be concentrated within an averaged minor radius of $\rho=0.2$ at $R_{\text {ax }}<3.55 \mathrm{~m}$. The power deposition profile is estimated with ray tracing including a weakly relativistic effect.

The NBI system was designed for achievement of high$n \tau T$ plasmas, where the target density is relatively high, more than $3 \times 10^{19} \mathrm{~m}^{-3}$. The nominal injection energy is as high as $180 \mathrm{keV}$ for the tangential hydrogen injection. ${ }^{23}$ Two negative-ion-based NB injectors of co- and counter(ctr)injection were operational in 1998, and one more ctr-injector was installed in $2001 .^{24}$ The co- and ctr-directions are defined here as the parallel ones to effective plasma currents which increase and decrease the rotational transform $(\iota)$, respectively. The total injection power achieved was $9 \mathrm{MW}$ with a hydrogen beam energy of around $160 \mathrm{keV}$. The power deposition profile is estimated with an orbit-following Monte Carlo calculation.

The profile measurements are performed for the transport analyses-YAG-laser Thomson scattering for the electron temperature $\left(T_{e}\right)$, charge-exchange recombination spectroscopy (CXRS) for the ion temperature $\left(T_{i}\right)$, and a multichord far-infrared (FIR) interferometer for the electron density $\left(n_{e}\right)$. Since the $T_{i}$-profile measurement is impossible in the magnetic field configuration of $R_{\mathrm{ax}}<3.75 \mathrm{~m}$ with CXRS, the $T_{i}$ profile is assumed parabolic with a central $T_{i}$ measured from a Doppler broadening of the TiXXI x-ray line.

\section{FORMATION OF ELECTRON INTERNAL TRANSPORT BARRIER}

In normal NBI-heated plasmas the electron temperature profile is fairly insensitive to the heating power and the density, and the core electron transport is dominated by the anomalous transport which could be described by the gyroBohm local heat conduction as $\chi_{e} \propto T_{e}^{3 / 2} .{ }^{25}$ When the centrally focused intense ECRH microwaves are injected to the plasma, a centrally peaked $T_{e}$ profile is observed, accompanying improvement of the core electron transport. Various shapes of the $T_{e}$ profile are obtained depending on the heating schemes such as ECRH+ctr-NBI, ECRH + co-NBI, and ECRH alone. The common feature is a steep gradient of the $T_{e}$ profile in a core plasma region, just like an internal transport barrier (ITB) observed in tokamaks. ${ }^{26}$ Thus, these profiles with the improvement of the electron transport observed in LHD are called electron-ITB here.

\section{A. Characteristics of electron-ITB}

In the case of superposition of the ECRH microwave on the ctr-injected NB-heated plasma, $T_{e}$ shows a centrally peaked ITB profile with a steep gradient inside $\rho=0.3-0.4$ at an electron density below a threshold one. Figure 1(a) shows an example of this $T_{e}$ profile, which has a foot-point defined as a location of the termination of the steep $T_{e}$ gradient. On the other hand, the electron-ITB profile observed in the case of the ECRH+co-NBI plasma is different from the 

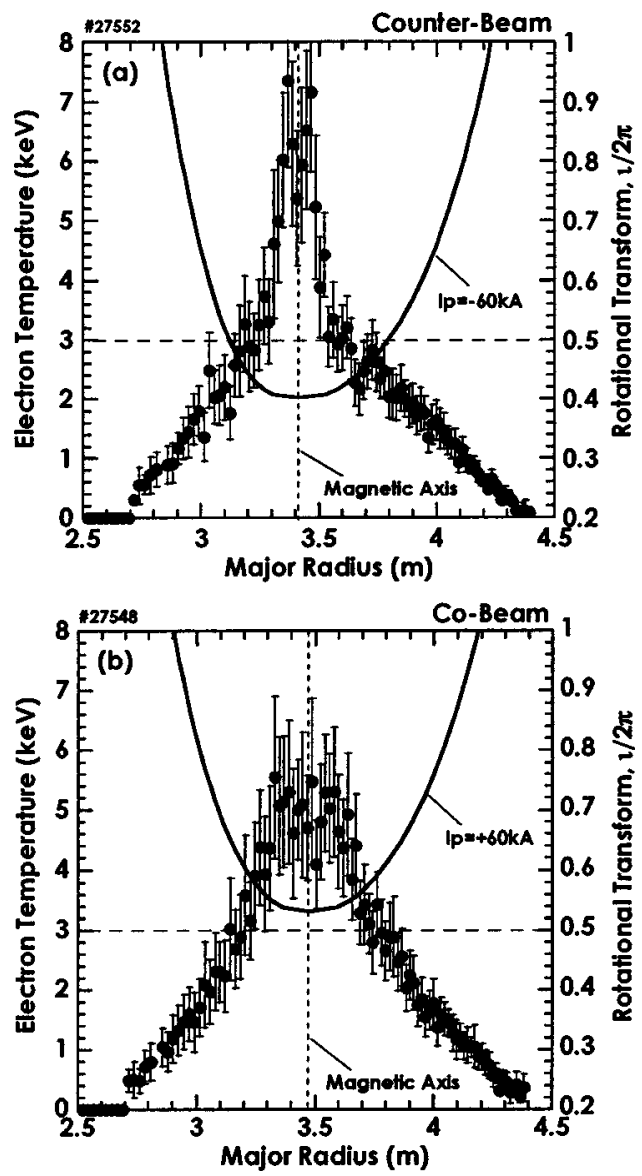

FIG. 1. Electron temperature profiles in the cases of superposition of the ECRH microwaves on (a) the counter-injected and (b) the co-injected NBheated plasmas. The rotational transform profiles $(\iota / 2 \pi)$ are also indicated with solid lines for the counter- and co-driven currents of -60 and $60 \mathrm{kA}$, respectively, together with the magnetic axis positions indicated by broken lines. The line-averaged electron density is $0.79 \times 10^{19} \mathrm{~m}^{-3}$ in (a) and $0.74 \times 10^{19} \mathrm{~m}^{-3}$ in (b), and the plasma current is $-42 \mathrm{kA}$ in (a) and $46 \mathrm{kA}$ in (b). $R_{\mathrm{ax}}=3.5 \mathrm{~m}$ and $B_{\mathrm{ax}}=2.854 \mathrm{~T}$.

$\mathrm{ECRH}+$ ctr-NBI one, as shown in Fig. 1(b), which is described in detail in the next section. There exist various kinds of thresholds for the electron-ITB formation, such as the ECRH power, the NBI power, and the density. Figure 2(a) shows the $T_{e}$ profiles for ECRH injection powers of 280, 180 and 0 (NBI-only) $\mathrm{kW}$ at $n_{e}=0.3 \times 10^{19} \mathrm{~m}^{-3}$ in the ECRH + ctr-NBI plasmas. The ECRH-power threshold is recognized for the electron-ITB formation. The electron transport analyses were performed for these profiles; one with an ITB, one without an ITB at a smaller ECRH power below the threshold, and one for an NBI-only plasma. The results are shown in Fig. 2(b). The $T_{e}$ is increased slightly and the electron thermal diffusivity, $\chi_{e}$, is much enhanced inside $\rho$ $=0.4$ when the smaller ECRH power $(180 \mathrm{~kW})$ is applied to the ctr-NBI plasma. When the ECRH power is increased $(280 \mathrm{~kW})$, the electron-ITB is formed with the central $T_{e}$ increased, and the $\chi_{e}$ is much reduced inside $\rho=0.4$. Figure 3(a) shows $\chi_{e}$ as a function of the collisionality normalized by $\nu_{\mathrm{eq}}=\varepsilon_{h}^{3 / 2} v_{T} \iota M / 2 \pi R$ at $\rho=0.2$ corresponding to Fig. 2 . Here, $\varepsilon_{h}$ and $\iota$ are the helical ripple and the rotational transform, respectively, $v_{T}$ is the electron thermal velocity, and $M$
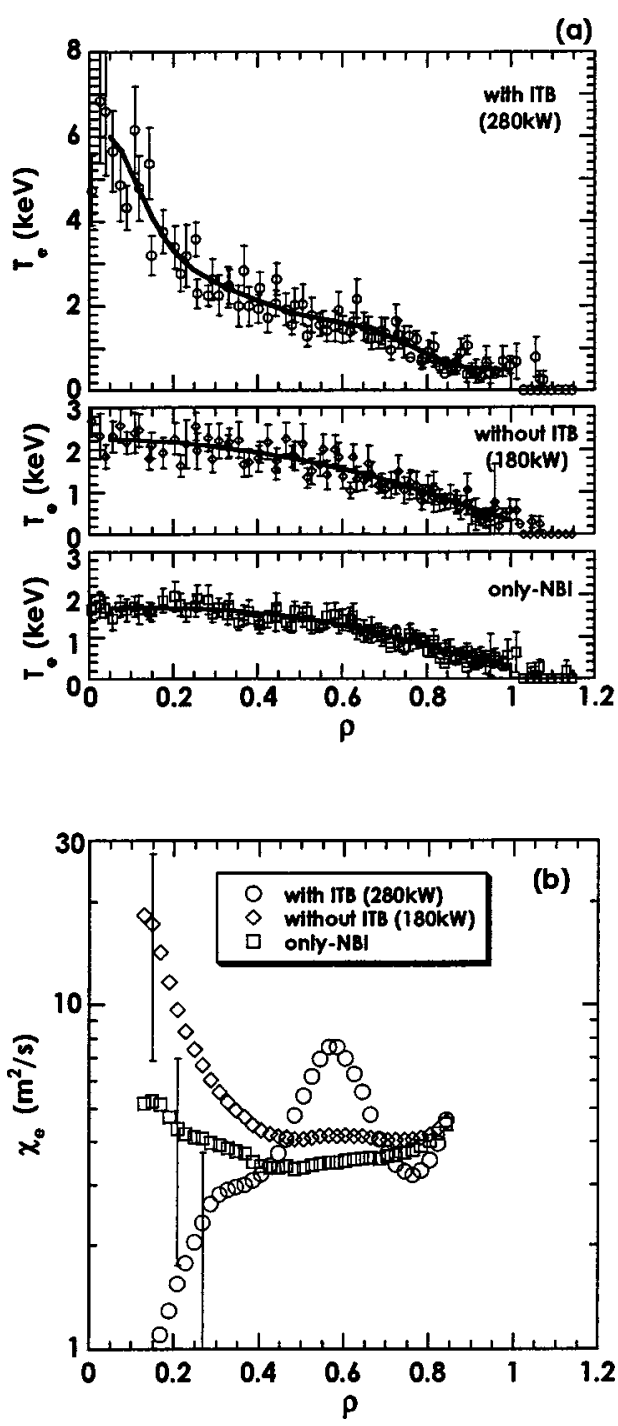

FIG. 2. (a) Electron temperature profiles with respect to the averaged minor radius, $\rho$, of the ECRH + counter-NBI plasmas with and without the ITB profile, and of the counter-NBI-only plasma. (b) Radial variation of the electron thermal diffusivity, $\chi_{e}$, for the three cases of plasmas in (a). Lineaveraged electron density is $0.3 \times 10^{19} \mathrm{~m}^{-3}$, and the ECRH injection power is 280 and $180 \mathrm{~kW}$ for the ITB and no-ITB plasmas, respectively.

and $R$ are the toroidal pitch number and the major radius, respectively. While $\chi_{e}$ is increased with a decrease in the normalized collisionality, $\nu_{b}^{*}$, due to an increase in $T_{e}$ for no ITB formation, it is abruptly reduced by about one order of magnitude for the ITB formation in spite of much increase in the $T_{e}$. The theoretically calculated radial electric field $\left(E_{r}\right)$ based on the neoclassical ambipolar flux shows the generation of a strong positive $E_{r}$ of $50 \mathrm{kV} / \mathrm{m}$ at around $\rho=0.2$ in the ITB formation while the $E_{r}$ is negative and small positive in the NBI-only and no-ITB plasmas, respectively, as shown in Fig. 3(b). This suggests the transition of the neoclassical ion root to the electron root, leading to the reduction of $\chi_{e}$ and the formation of the electron-ITB. Measurement with the CXRS, which is made at $R_{\mathrm{ax}}=3.75 \mathrm{~m}$ and $B_{\mathrm{ax}}=1.52 \mathrm{~T}$ due to constraint on the measurable region and the EC resonant magnetic field strength, shows a positive $E_{r}$ of about 10 $\mathrm{kV} / \mathrm{m}$ at $\rho=0.25$. $^{27}$ The electron transport in no-ITB plasmas 

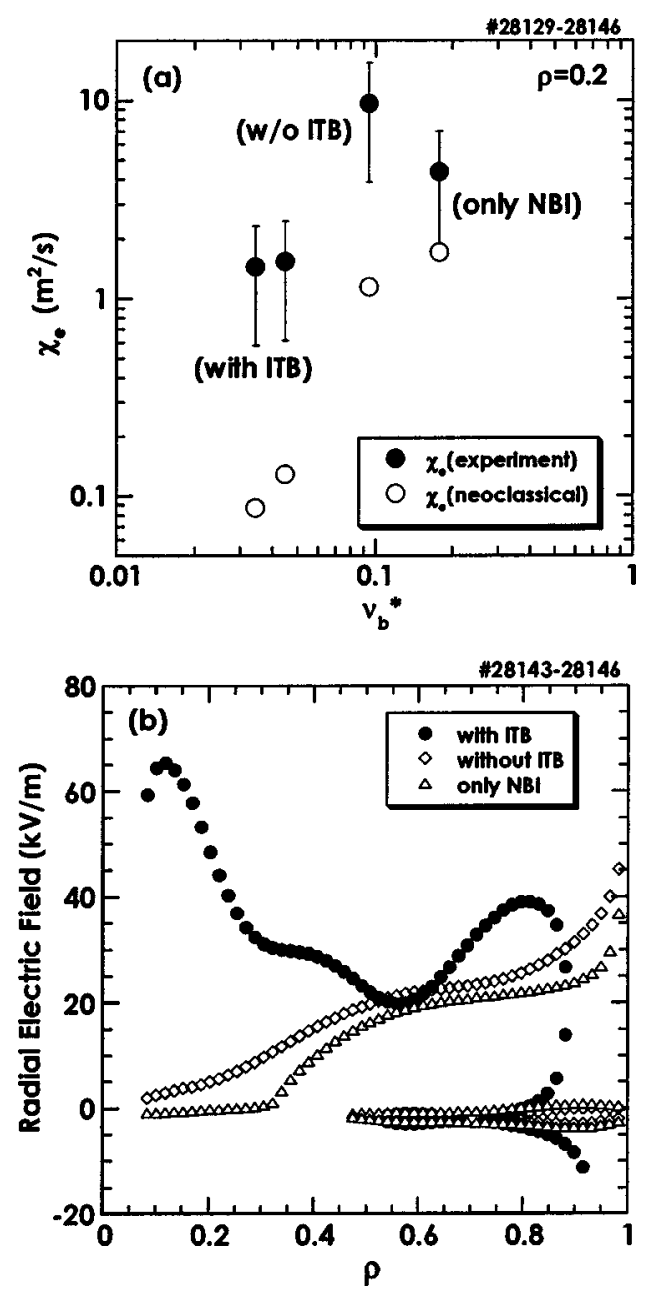

FIG. 3. (a) Electron thermal diffusivity, $\chi_{e}$, as a function of the normalized collisionality, $\nu_{b}^{*}$, which is the collisionality normalized by $\nu_{\mathrm{eq}}=\varepsilon_{h}{ }^{3 / 2} v_{T} \iota$ $M / 2 \pi R$ at $\rho=0.2\left(\varepsilon_{h}\right.$ : helical ripple, $\iota$ : rotational transform, $v_{T}$ : electron thermal velocity, $M$ : toroidal pitch number, and $R$ : major radius). (b) Radial electric field profiles calculated theoretically based on the neoclassical ambipolar flux for plasmas in Fig. 2.

should be dominated by the anomalous transport where the degree of anomalousness is enhanced with an increase in the $T_{e}$. The electron root with the positive $E_{r}$ should strongly suppress the enhancement of the anomalous transport due to the $T_{e}$ rise. However, the $\chi_{e}$ in the electron ITB is one order of magnitude larger than the theoretical one in the neoclassical electron root, as shown in Fig. 3(a).

\section{B. Influence of injection direction of neutral beams}

As shown in Fig. 1(b), the $T_{e}$ profile in the case of the $\mathrm{ECRH}+$ co-injected NBI plasma has no distinct foot-point and the central $T_{e}$ is a little lower although the region of steep gradient is wider, compared with the ECRH+ctr-NBI plasma. The plasma axis is shifted by the plasma and beam pressure (Shafranov shift), and the rotational transform profile is changed. The co-driven current shifts the plasma axis outward while the counter-driven one shifts it inward for the zero-beta plasma at $R_{\mathrm{ax}}=3.5 \mathrm{~m}$. In the experimental condition in Fig. 1, the ECRH resonance position with respect to $\rho$ deviates little from the magnetic axis for the co-injected

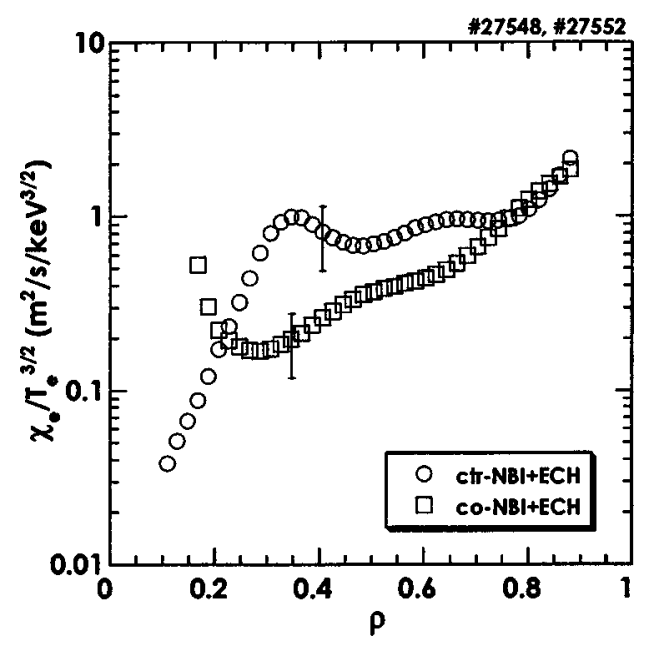

FIG. 4. Electron thermal diffusivity, $\chi_{e}$, normalized by $T_{e}^{3 / 2}$ of the gyroBohm factor for the ECRH plasmas superposed on the co- and counterinjected NBI ones in Fig. 1.

plasma, compared with the ctr-injected one. The rotational transform profiles are also indicated in Figs. 1(a) and 1(b) for ctr- and co-driven currents of $60 \mathrm{kA}$, respectively. The footpoint location of the ITB profile in Fig. 1(a) seems to correlate with the position of the rational surface of $\iota / 2 \pi=0.5$, which disappears in the co-injected plasma. Although the location and the size of the magnetic island of $\iota / 2 \pi=0.5$ including the beam pressure effect is not calculated, they should be sensitive to the beam-driven current including the injection direction. The parallel transport along the magnetic flux surface is much more dominant than the cross-field transport in the island, and a flattening of the $T_{e}$ profile could be observed around the island. In the tangential view of the two-dimensional soft $x$-ray camera, local flattening corresponding to the $\iota / 2 \pi=0.5$ island was observed, and its location coincided roughly with that of the $T_{e}$ flattening. ${ }^{28}$ The $T_{e}$ flattening related to the island also corresponds to the large $\chi_{e}$ around $\rho=0.57$, where $\iota / 2 \pi=0.52$, in Fig. 2(b). Considering the properties related to the magnetic island, the foot-point corresponding to the termination of the steep $T_{e}$ gradient in the ITB profile is ascribed to the location of the $\iota / 2 \pi=0.5$ magnetic island. Therefore, in the co-injected plasma no distinct foot-point is observed although the $T_{e}$ gradient around $\rho=0.2$ is almost comparable to that in the counter-injected ITB plasma.

Figure 4 shows a result of the transport analysis for the co- and counter-injected plasmas in Fig. 1. The $\chi_{e}$ normalized by $T_{e}^{3 / 2}$ of the gyro-Bohm factor is used for estimation of the degree of improvement of the anomalous transport. The normalized $\chi_{e}$ is reduced toward the plasma center in a wide region for the co-injected plasma while it is rapidly reduced inside $\rho=0.3$ and is nearly constant outside $\rho=0.3$ for the counter-injected one. It is found that the transport improvement is recognized in a wider region for the coinjected plasma. On the other hand, for the counter-injected plasma, inside $\rho=0.2$ the normalized $\chi_{e}$ is reduced largely compared with that for the co-injected one. At a local transition boundary to the electron root from the ion root, corresponding to a region just inside the foot-point, a strong $E_{r}$ 

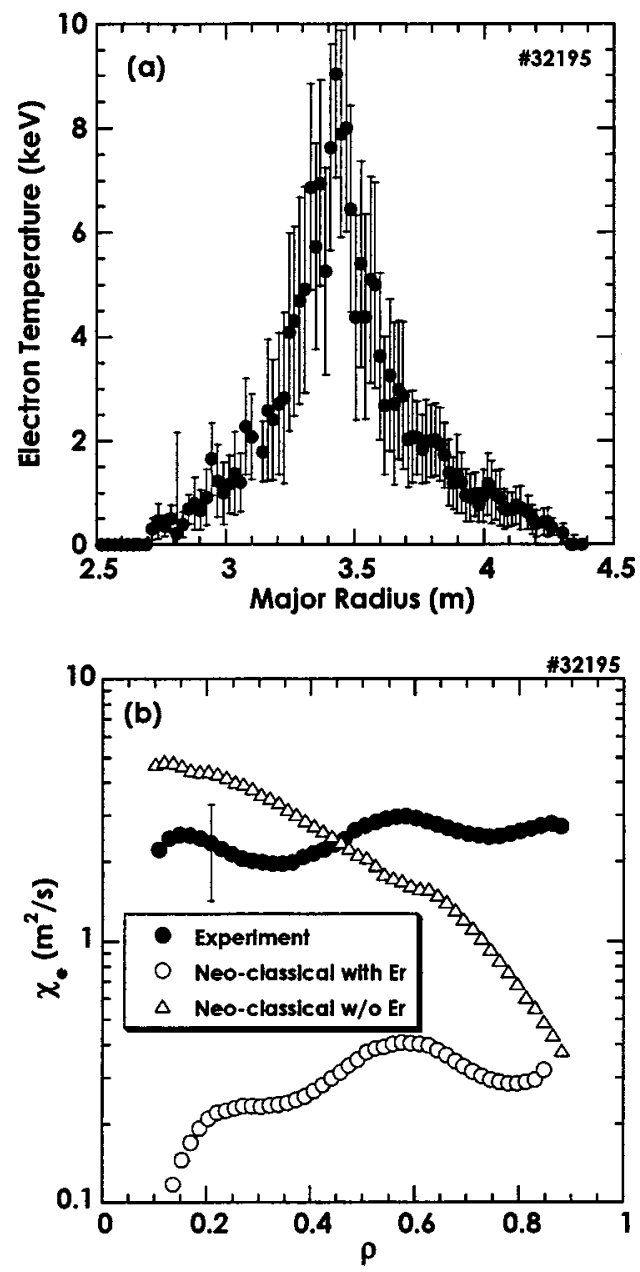

FIG. 5. (a) Electron temperature profile of the ECRH-only plasma. The line-averaged electron density is $0.5 \times 10^{19} \mathrm{~m}^{-3}, R_{\mathrm{ax}}=3.5 \mathrm{~m}$, and $B_{\mathrm{ax}}$ $=2.854 \mathrm{~T}$. (b) Electron thermal diffusivity, $\chi_{e}$, estimated with the transport analysis for the plasma of (a) together with the neoclassical $\chi_{e}$ considering the radial electric field estimated with the neoclassical ambipolar flux and without assuming the radial electric field.

shear could be formed, which is expected to strongly suppress the anomalous transport. In the theoretical calculation the $E_{r}$ shear is formed at the $E_{r}$ transition boundary, $\rho$ $<0.5$, in the ctr-injected plasma as shown in Fig. 3(b). For the co-injected plasma without a distinct foot-point, the local transition should be weak even if it exists, and strong $E_{r}$ shear should not develop.

\section{Comparison with ECRH plasmas}

The $T_{e}$ profile shape of the ECRH-only plasma is different from the ECRH+NBI plasma in the same $R_{\text {ax }}(3.5 \mathrm{~m})$ and $B_{\text {ax }}(2.854 \mathrm{~T})$. Figure 5(a) shows a typical $T_{e}$ profile of the ECRH plasma, which is a centrally peaked profile with a steep and concave gradient. Although the central $T_{e}$ changes from over 10 to $2 \mathrm{keV}$ depending on the electron density, the profile shape is rather invariant and no foot-point is observed. Also, no threshold is observed such as in the ECRH power, the density, and the collisionality. $\chi_{e}$ estimated with the transport analysis is shown in Fig. 5(b) for the $T_{e}$ profile in Fig. 5(a) together with the neoclassical $\chi_{e}$ considering the $E_{r}$ estimated with the neoclassical ambipolar flux. For com-

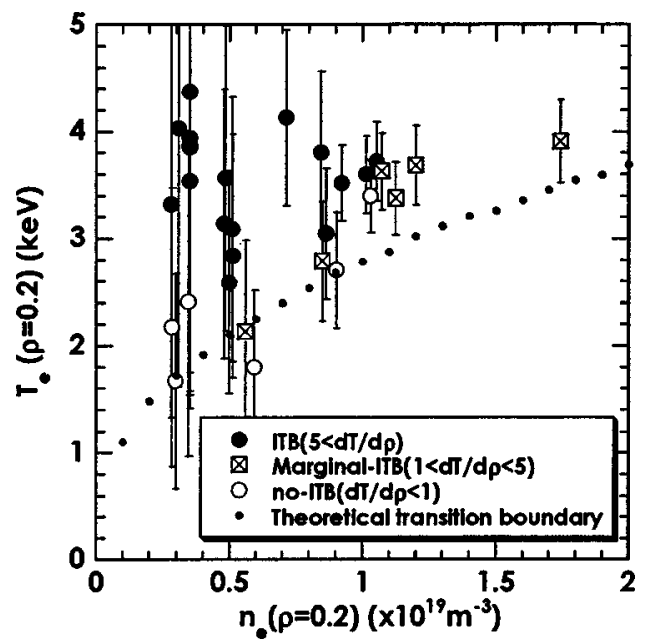

FIG. 6. $T_{e}-n_{e}$ diagram for both ITB and no-ITB profiles at $\rho=0.2$ for the $\mathrm{ECRH}+\mathrm{NBI}$ plasmas. The ITB formation is classified by the gradient of $T_{e}, d T_{e} / d \rho$, as follows; no ITB formation for $d T_{e} / d \rho<1 \mathrm{keV}$, ITB formation for $d T_{e} / d \rho>5 \mathrm{keV}$, and marginal ITB formation for $1 \mathrm{keV}$ $<d T_{e} / d \rho<5 \mathrm{keV}$. The theoretical transition boundary to the electron root (positive $E_{r}$ ) from the ion root (weak positive or negative $E_{r}$ ) is also indicated by a dotted line, assuming $T_{e} / T_{i}=2$.

parison, the neoclassical $\chi_{e}$ without assuming $E_{r}$ is also plotted in the figure. The experimental $\chi_{e}$ has almost no radial dependence in spite of the large variation of the $T_{e}$, and it is comparable to that of the ECRH + ctr-NBI plasma at $\rho$ $=0.2$. Considering that the calculated $E_{r}$ is about $50 \mathrm{kV} / \mathrm{m}$ at $\rho=0.2$, the improvement of the $\chi_{e}$ in the electron root is recognized, although the experimental $\chi_{e}$ is still larger by one order of magnitude than the neoclassical $\chi_{e}$ including the $E_{r}$. As the experimental $\chi_{e}$ is smaller than the neoclassical $\chi_{e}$ without the $E_{r}$ in the core region, the enhanced transport in the collisionless region due to the asymmetry could be reduced by the $E_{r}$.

\section{Transition to electron root}

For the ITB formation in the ECRH+ NBI plasma there exist parametrical thresholds, such as the heating power and the density, and a spatial threshold observed as the footpoint. Above the threshold, the electron transport is reduced and the $T_{e}$ is increased, which could be ascribed to the $E_{r}$ generated by the neoclassical ambipolar flux. Therefore, the threshold should be correlated with the transition to the strong positive $E_{r}$, meaning the transition to the neoclassical electron root. The local ITB formation at a radial position is judged by the gradient of $T_{e}, d T_{e} / d \rho$, and the following are defined: no ITB formation for $d T_{e} / d \rho<1 \mathrm{keV}$, ITB formation for $d T_{e} / d \rho>5 \mathrm{keV}$, and marginal ITB formation for $1 \mathrm{keV}<d T_{e} / d \rho<5 \mathrm{keV}$. Figure 6 shows the $T_{e}-n_{e}$ diagram for both ITB and no-ITB profiles at $\rho=0.2$. In Fig. 6 , the theoretical transition boundary to the electron root (positive $E_{r}$ ) from the ion root (weak positive or negative $E_{r}$ ) is also indicated by a dotted line, assuming $T_{e} / T_{i}=2 .{ }^{29}$ As shown in Fig. 6, for the ECRH+NBI plasmas the temperature threshold for the ITB formation is dependent on $n_{e}^{0.4}$, which is consistent with the theoretical prediction. The electron ITB profile is observed also in relatively high-density plasmas of 


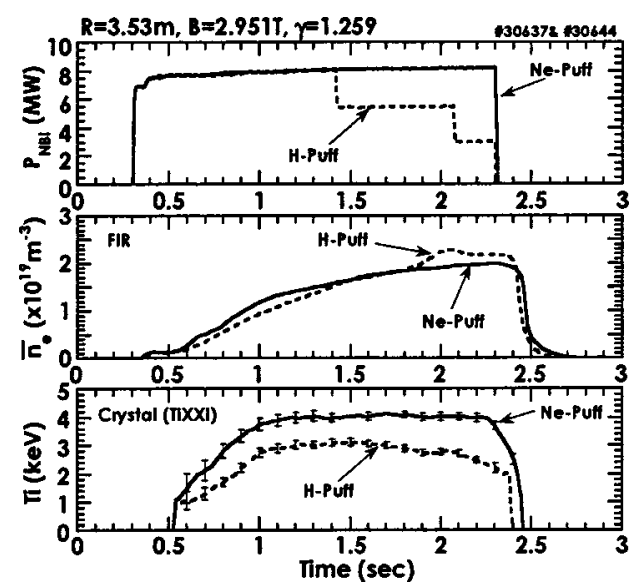

FIG. 7. Time evolutions of the injected NBI power, $P_{\mathrm{NBI}}$, the line-averaged electron density, $n_{e}$, and the central ion temperature measured with the Doppler broadening of the TiXXI, $T_{i}$, for the neon-injected plasma and the hydrogen one in $R_{\mathrm{ax}}=3.53 \mathrm{~m}$.

$>1 \times 10^{19} \mathrm{~m}^{-3}$ with a higher ECRH power and a higher NBI power. The transition to the electron root is apparently recognized in the ECRH + ctr-NBI plasmas while that seems to be gradual in the ECRH+co-NBI ones. Judging from the theoretical $E_{r}$ calculation result using experimental profiles, as shown in Fig. 3(b), the local transition of the $E_{r}$ seems to occur at around $\rho=0.5$, which corresponds to the foot-point position. For the ECRH+ co-NBI plasma, however, this local transition is not distinguished clearly. As for the ECRH-only plasmas, no distinct transition exists for both the plasma parameter and the local position, and the ITB is formed for lower $T_{e}$ plasmas with a higher density than the ECRH + NBI plasmas. The ECRH-only plasmas could be in the electron root with a positive $E_{r}$ at the start of the discharge, and, thus, the transition itself could never occur. Although a clear increase in the ion temperature has not been observed, a high electron temperature in the ITB formation should enhance the ion-heating ratio in high-power NBI heating, leading to an increase in the ion temperature. Moreover, the neoclassical electron root in the ITB formation should reduce the ion transport in the collisionless regime, which could realize a higher ion temperature.

\section{ACHIEVEMENT OF HIGH ION TEMPERATURE}

The hydrogen beam injection energy is as high as over $150 \mathrm{keV}$, aiming to achieve a high- $n \tau T$ plasma with a density of $(3-4) \times 10^{19} \mathrm{~m}^{-3}$. Therefore, the plasma heating power is much reduced for low-density plasmas below 1 $\times 10^{19} \mathrm{~m}^{-3}$ due to a large amount of the shine-through of the injected neutral beam. Moreover, the injection energy is so high that the electron heating power should be dominant, and the achieved ion temperature is usually less than $2.5 \mathrm{keV}$ in hydrogen plasmas. ${ }^{30}$ In order to increase the plasma absorption power of the high-energy neutral beams in low-density plasmas, neon gas was injected, instead of hydrogen or helium gas. Figure 7 shows the comparison of the neoninjected plasma with the hydrogen one in $R_{\mathrm{ax}}=3.53 \mathrm{~m}$. Although the port-through NBI power is almost the same, around $8 \mathrm{MW}$, in both cases, the ion temperature in the neon

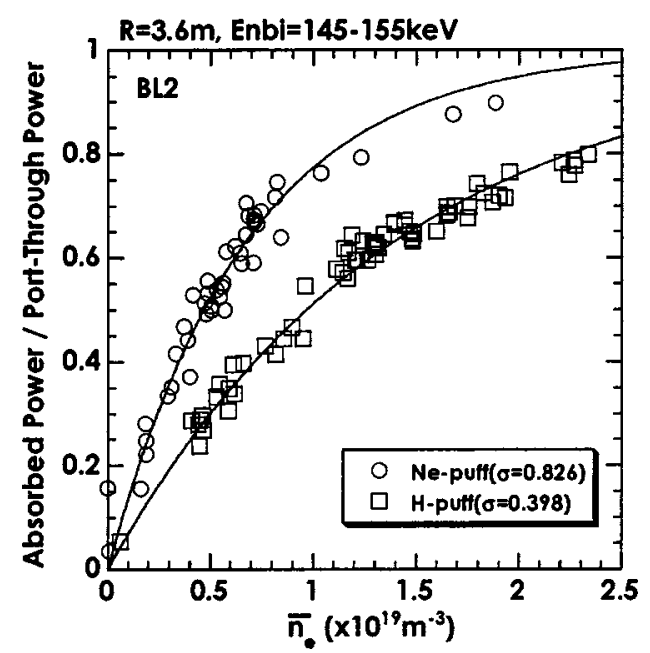

FIG. 8. Ratio of the absorbed power to the port-through power of the injected neutral beams as a function of the line-averaged electron density, $n_{e}$, for the neon- and hydrogen-puffed plasmas in $R_{\mathrm{ax}}=3.6 \mathrm{~m}$. The injection beam energy is $145-155 \mathrm{keV}$.

plasma is higher by about $1 \mathrm{keV}$ than that in the hydrogen plasma at the same density of $n_{e}=(1-2) \times 10^{19} \mathrm{~m}^{-3}$. The ionized beam power (plasma absorption power) is estimated from the shine-through power measurement, ${ }^{31}$ and the ratio of the absorption power $\left(P_{\text {abs }}\right)$ to the port-through power $\left(P_{\text {port }}\right)$ is shown in Fig. 8 for both neon- and hydrogenpuffed plasmas. The beam ionization rate is much higher in the neon plasmas than that in the hydrogen ones, and the plasma absorption power is about 1.5 times larger in the neon plasma at $n_{e}=1 \times 10^{19} \mathrm{~m}^{-3}$. When the $P_{\text {abs }} / P_{\text {port }}$ is fitted to $1-\exp (-\sigma n l), \sigma$ is thought to be the effective ionization cross section for the injected neutral beam. Here, $n l$ is the line-integrated electron density along the FIR central cord. Since $\sigma$ is proportional to the effective ionic charge $\left(Z_{\text {eff }}\right)$ and the $\sigma$ in the neon plasma is about two times larger than that in the hydrogen plasma, the $Z_{\text {eff }}$ in the neon plasma should be roughly estimated at around 6 , considering that the $Z_{\text {eff }}$ of the hydrogen plasmas is around 3. Assuming that the plasma ion species are only neon and hydrogen, the ion density is nearly half of the electron density. Therefore, the heating power to the ions is much increased in the neon plasmas. Figure 9 shows the central ion temperature as a function of the plasma absorption power normalized by the lineaveraged electron density for both neon and hydrogen plasmas. Since the electron temperature is not so different between them, the heating power rate to one ion for the neon plasmas is double that for the hydrogen plasmas. As shown in Fig. 9, the ion temperature in the neon plasmas is increased linearly to the normalized absorption power and reaches $5 \mathrm{keV}$, while it is lower and is saturated in higher powers in the hydrogen plasmas.

The transport analyses of the neon plasmas show that the electron transport is not largely changed compared with the hydrogen or helium plasmas. ${ }^{32}$ At $R_{\mathrm{ax}}=3.6 \mathrm{~m}$ the ion temperature profiles at outer radii can be measured with the CXRS, and they are shown in Fig. 10 together with the $T_{e}$ profiles for both neon and helium plasmas. Although the $T_{i}$ profile at outer radii $(\rho>0.6)$ in the neon plasma is nearly 


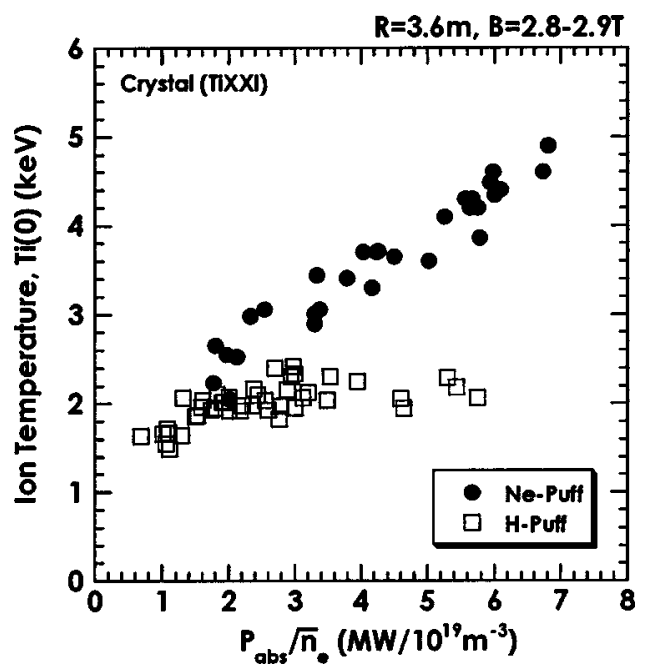

FIG. 9. Central ion temperature, $T_{i}(0)$, as a function of the plasma absorption power normalized by the line-averaged electron density, $P_{\text {abs }} / n_{e}$, for the neon and hydrogen plasmas in $R_{\mathrm{ax}}=3.6 \mathrm{~m}$.
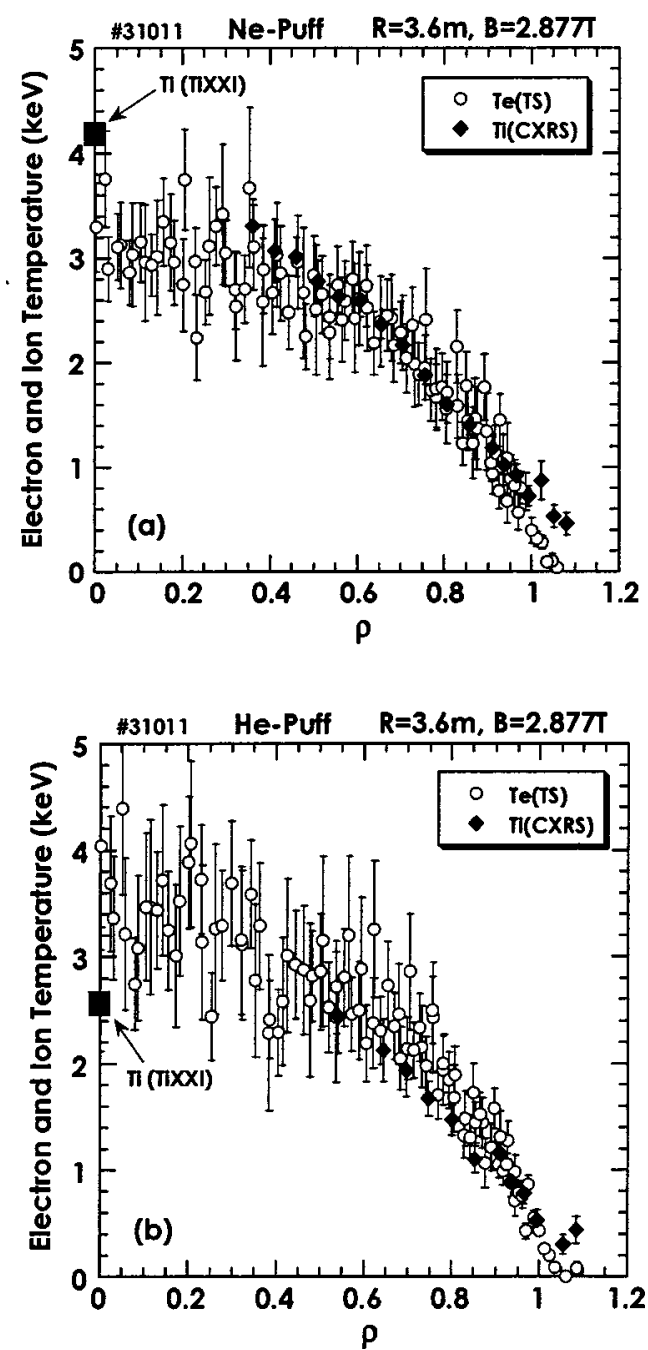

FIG. 10. Ion temperature profiles measured with the CXRS at outer radii ( ) and with the Doppler broadening of TiXXI at center $(\boldsymbol{\square})$, together with the electron temperature profiles $(\mathrm{O})$, for $(\mathrm{a})$ the neon- and (b) the heliumpuffed plasmas. $R_{\mathrm{ax}}=3.6 \mathrm{~m}$ and $B_{\mathrm{ax}}=2.877 \mathrm{~T}$. the same as or a little higher than that in the helium plasma, the central $T_{i}$ measured with the TiXXI doppler broadening is higher by $1.5 \mathrm{keV}$ in the neon plasma. This suggests that the $T_{i}$ profile at inner radii $(\rho<0.5)$ is largely changed by the neon injection. Considering that the central $T_{i}$ is saturated at a higher $P_{\text {abs }} / n_{e}$ for the hydrogen plasma while the $T_{i}$ saturation is not so strong for the neon one, it is found that an anomalous increase of the ion transport with an increase in the heating power is not observed in the neon plasma. The collisionality of neon ions is as large as in the plateau regime because the ion collisionality depends on $Z^{3}$. The ion thermal diffusivity, roughly estimated by assuming that the $T_{i}$ profile is parabolic, is $2-5 \mathrm{~m}^{2} / \mathrm{s}$ in the neon plasmas, and is nearly the same as or a little larger than that in the helium or hydrogen plasmas of which the collisionality is smaller by one to two orders of magnitude.

Although the effect of the neon injection can be explained by an increase in the plasma absorption power and enhancement of the direct ion heating power with a reduced number of ions, it is found that the central $T_{i}$ is simply raised by an increase in the ion heating power.

\section{CONCLUDING REMARKS}

In LHD, the ITB is formed for the electron temperature profile with centrally focused ECRH microwaves injected into the plasma. A high $T_{e}$, exceeding $10 \mathrm{keV}$ in a low density, is achieved by improvement of the core electron transport. The electron-ITB observed in LHD is characterized by the neoclassical electron root with a positive radial electric field determined by the neoclassical ambipolar flux. In the case of the ECRH+NBI plasma, the $T_{e}$ threshold for the ITB formation depends on the $n_{e}^{0.4}$, which is consistent with the theoretically predicted transition to the electron root with a strong positive $E_{r}$. The $E_{r}$ transition was also observed experimentally. The $m / n=2 / 1$ magnetic island has an influence on the radial position of the transition boundary, which is observed as a foot-point of the ITB. The ITB profile is narrow with a steep gradient inside $\rho=0.4$ for the ECRH + ctr-NBI plasma while that is broader without the foot-point for the ECRH+co-NBI plasma. The beam-driven current modifies the rotational transform profile and changes the location and the size of the $m / n=2 / 1$ island, over which the transport is large. The counter-directed beam flow should enhance the island structure causing the deteriorated transport. As a result, just inside the foot-point, a strong $E_{r}$ shear develops, leading to the steeper gradient with a reduction of the anomalous transport.

As for the ECRH-only plasma, although no distinct transition is observed, an electron-ITB profile is also formed and a positive $E_{r}$ is estimated by the theoretical calculation. The electron thermal transport is lower than that of the neoclassical one without considering the $E_{r}$ effect, suggesting that the enhanced ripple transport in the collisionless regime should be suppressed by the radial electric field, although the $\chi_{e}$ is still larger by an order of magnitude than the neoclassical value including the $E_{r}$ effect.

A high-ion temperature of $5 \mathrm{keV}$ was achieved with neon injection. The net heating power to the ions is much en- 
hanced by both an increase in the plasma absorption power of the injected beam and a reduction of the ion number. It is encouraging that the ion temperature is increased with an increase in the net heating power to the ions, and a further increase in the NBI power is required. In the neon plasmas a marginal electron ITB profile is observed at a relatively high density of over $1.5 \times 10^{19} \mathrm{~m}^{-3}$. It is important to realize a high ion temperature in the neoclassical electron root, where the ion transport is improved.

\section{ACKNOWLEDGMENTS}

The authors are grateful to world-wide collaborations, and collaborational efforts to perform the LHD project by the Japanese universities and other institutes are greatly appreciated. The authors would like to gratefully acknowledge the continuous efforts of the engineering and technical staff to the LHD operation.

${ }^{1}$ O. Motojima, N. Yanagi, S. Imagawa et al., Proceedings of the 16th International Conference on Fusion Energy, Montreal, 1996 (International Atomic Energy Agency, Vienna, 1997), Vol. 3, p. 437.

${ }^{2}$ A. Iiyoshi, A. Komori, A. Ejiri et al., Nucl. Fusion 39, 1245 (1999).

${ }^{3}$ O. Motojima, H. Yamada, A. Komori et al., Phys. Plasmas 6, 1843 (1999).

${ }^{4}$ M. Fujiwara, K. Kawahata, N. Ohyabu et al., Nucl. Fusion 41, 1355 (2001).

${ }^{5}$ U. Stroth, M. Murakami, H. Yamada et al., Nucl. Fusion 36, 1063 (1996).

${ }^{6}$ H. Yamada, K. Y. Watanabe, S. Sakakibara et al., Phys. Rev. Lett. 84, 1216 (2000).

${ }^{7}$ N. Ohyabu, K. Narihara, H. Funaba et al., Phys. Rev. Lett. 84, 103 (2000).

${ }^{8}$ S. Sakakibara, H. Yamada, K. Watanabe et al., Nucl. Fusion 41, 1177 (2001).

${ }^{9}$ H. Yamada, A. Komori, N. Ohyabu et al., Plasma Phys. Controlled Fusion 43, A55 (2001).

${ }^{10}$ T. Mutoh, R. Kumazawa, T. Seki et al., Phys. Rev. Lett. 85, 4530 (2000).

${ }^{11}$ T. Watari, T. Mutoh, R. Kumazawa et al., Nucl. Fusion 41, 325 (2001).

${ }^{12}$ R. Kumazawa, T. Mutoh, T. Seki et al., Phys. Plasmas 8, 2139 (2001).

${ }^{13}$ Y. Takeiri, Y. Nakamura, N. Noda et al., Plasma Phys. Controlled Fusion 42, 147 (2000).

${ }^{14}$ M. Fujiwara, Y. Takeiri, T. Shimozuma et al., Nucl. Fusion 40, 1157 (2000).

${ }^{15}$ Y. Nakamura, Y. Takeiri, R. Kumazawa et al., "Plasma performance and impurity behavior in long pulse discharges on LHD," in Proceedings of the 19th Fusion Energy Conference, Lyon, 2002 (International Atomic Energy Agency, Vienna, to be published), EX/C5-1.

${ }^{16}$ H. Maassberg, C. D. Beidler, U. Gasparino et al., Phys. Plasmas 7, 295 (2000).

${ }^{17}$ A. Fujisawa, H. Iguchi, T. Minami et al., Phys. Rev. Lett. 82, 2669 (1999).

${ }^{18}$ S. Kubo, T. Shimozuma, H. Idei et al., J. Plasma Fusion Res. 78, 99 (2002).

${ }^{19}$ T. Shimozuma, S. Kubo, H. Idei et al., "Formation of electron internal transport barrier by highly localized electron-cyclotron-resonance heating in the Large Helical Device,"'" Plasma Phys. Controlled Fusion (submitted).

${ }^{20}$ S. Morita, M. Goto, Y. Takeiri et al., "Study on ion temperature behaviors in electron and ion heating regimes of ECH, ICRF and NBI discharges in LHD," in Proceedings of the 19th Fusion Energy Conference, Lyon, 2002 (International Atomic Energy Agency, Vienna, to be published), EX/P2-18.

${ }^{21}$ Y. Takeiri, M. Osakabe, K. Ikeda et al., Proceedings of the 29th European Physical Society Conference on Plasma Physics and Controlled Fusion, Montreux, 2002 (European Physical Society, Petit-Lancy, 2002), P-1.076.

${ }^{22}$ T. Shimozuma, S. Kubo, M. Sato et al., Fusion Eng. Des. 53, 525 (2001).

${ }^{23}$ Y. Takeiri, O. Kaneko, Y. Oka et al., Proceedings of the 17th IEEE/NPSS Symposium on Fusion Engineering, San Diego, 1997 (Institute of Electrical and Electronics Engineers, New York, 1998), Vol. 1, p. 409.

${ }^{24} \mathrm{O}$. Kaneko, Y. Takeiri, K. Tsumori et al., "Engineering prospects of negative-ion-based neutral injection system from high power operation for the Large Helical Device," in Proceedings of the 19th Fusion Energy Conference, Lyon, 2002 (International Atomic Energy Agency, Vienna, to be published), CT-6Rb.

${ }^{25} \mathrm{H}$. Yamada, S. Murakami, K. Yamazaki et al., "Response of temperature and density profiles to heat deposition profile and its impact on global scaling in LHD," Proceedings of the 19th Fusion Energy Conference, Lyon, 2002 (International Atomic Energy Agency, Vienna, to be published), EX/C4-5Ra.

${ }^{26}$ T. Fujita, S. Ide, H. Shirai et al., Phys. Rev. Lett. 78, 2377 (1997).

${ }^{27}$ K. Ida, H. Funaba, K. Narihara et al., "Radial electric field and electron heat transport of the plasma with electron internal transport barrier in Large Helical Device," Phys. Rev. Lett. (submitted).

${ }^{28}$ Y. Liang, K. Ida, K. Y. Watanabe et al., "Effect of $n / m=1 / 2$ magnetic island on transport in the plasma with neoclassical internal transport barrier in the Large Helical Device," Phys. Plasmas (submitted).

${ }^{29}$ M. Yokoyama, K. Ida, H. Sanuki et al., Nucl. Fusion 42, 143 (2002).

${ }^{30}$ S. Morita, M. Goto, S. Kubo et al., Nucl. Fusion 42, 1179 (2002).

${ }^{31}$ M. Osakabe, Y. Takeiri, K. Tsumori et al., Rev. Sci. Instrum. 72, 590 (2001).

${ }^{32}$ J. Miyazawa, H. Yamada, S. Morita et al., Proceedings of the 29th European Physical Society Conference on Plasma Physics and Controlled Fusion, Montreux, 2002 (European Physical Society, Petit-Lancy, 2002), P-1.073. 